\title{
Excitations in confined helium
}

\author{
V. Apaja ${ }^{1,2}$ and E. Krotscheck ${ }^{1}$ \\ ${ }^{1}$ Institut für Theoretische Physik, Johannes Kepler Universität, A 4040 Linz, Austria and \\ ${ }^{2}$ Department of Physical Sciences, Theoretical Physics, University of Oulu, FIN-90014 Oulu, Finland
}

\begin{abstract}
We design models for helium in matrices like aerogel, Vycor or Geltech from a manifestly microscopic point of view. For that purpose, we calculate the dynamic structure function of ${ }^{4} \mathrm{He}$ on $\mathrm{Si}$ substrates and between two Si walls as a function of energy, momentum transfer, and the scattering angle. The angle-averaged results are in good agreement with the neutron scattering data; the remaining differences can be attributed to the simplified model used here for the complex pore structure of the materials. A focus of the present work is the detailed identification of coexisting layer modes and bulk-like excitations, and, in the case of thick films, ripplon excitations. Involving essentially two-dimensional motion of atoms, the layer modes are sensitive to the scattering angle.
\end{abstract}

\section{INTRODUCTION}

There is much current interest in understanding the properties of superfluid ${ }^{4} \mathrm{He}$ in confinement. In particular, collective excitations of superfluid helium confined to silica aerogel have been studied by neutron scattering since the early 90's [1, 2], and by now a wealth of information about helium in aerogel, Vycor and Geltech has been collected [3, 4, 5, 6, 7, 8, 9, 10, 11]. Aerogel is an open gel structure formed by silica strands $\left(\mathrm{SiO}_{2}\right)$. Typical pore sizes range from few $\AA$ to few hundred $\AA$, without any characteristic pore size. Vycor is a porous glass, where pores form channels of about $70 \AA$ diameter. Geltech resembles aerogel, except that the nominal pore size is $25 \AA$ 11]. When liquid helium is placed in such an environment, it will first be physisorbed to the free surfaces of the matrices. Such an adsorption occurs in layers, the first layer of ${ }^{4} \mathrm{He}$ is expected to be solid; on a more strongly binding substrate, such as graphite, one expects two solid layers. In this work we are restricted to the liquid state, and the layer of solid helium is considered as part of the substrate.

Recent very accurate instruments have, contrary to earlier findings, revealed that the energies and lifetimes of phonon-roton excitations for confined ${ }^{4} \mathrm{He}$ are nearly equal to their bulk superfluid ${ }^{4} \mathrm{He}$ values. Specifically, the roton linewidth was found by Anderson et al. 12] to be less than the instrumental resolution, $0.1 \mu \mathrm{m}$. As expected, differences usually appear at partial fillings. The appearance of ripplons is tied to the existence of a free liquid surface; neutron scattering experiments show clearly their presence in on adsorbed films 13, 14 and in aerogel containing few layers of helium [15, 16].

Since nothing spectacular could be seen in the bulklike mode, the focus shifted to nearly two-dimensional "layer modes". The existence of collective excitations below the roton minimum has been demonstrated first by Lauter and collaborators 17, 18, 19, 20]; these modes were identified as longitudinal phonons propagating in the first liquid layer close to the substrate [21, 22, 23]. The nature of these modes has been found to be very resilient and quite independent on the substrate strength [24], only for very weakly attractive substrates like Cs, the "layer phonons" acquire a transverse component and become similar to ripplons. Layer modes in helium have been observed in Vycor 25 and in both fully filled and partially filled aerogel above wave vector $1.7 \AA^{-1}$ [26]. Therefore, layer modes are the only excitations observed that are characteristic for helium films or helium in aerogel or Vycor.

The effect of disorder on macroscopic properties of both ${ }^{3} \mathrm{He}$ and ${ }^{4} \mathrm{He}$ has been investigated in detail, but there are only few theoretical studies on excitations that apply to confined helium. Locally, the density profile of the liquid can be assumed to be that of a helium film 27] or helium filling a space between two planes [28]. Impurity induced disorder has been studied using Path Integral Monte Carlo 29]. At the moment there are no close-to-reality models of the confining structures combined with a proper theory of superfluid helium. Obvious simplified model systems are liquid helium filling random voids or helium between parallel planes. The former is computationally very demanding. Experiments have covered the full range of helium thicknesses, from a bare monolayer to a filled sample, and the results show that layer modes are visible against the background of bulk excitations if there are about four or five layers of helium; in those cases we are basically dealing with a thick He film on Si. However, the pores in aerogel and Geltech are not filling uniformly, which has lead to the notion of connected superfluidity and localized Bose-Einstein condensation [1]. Thus a well-defined phonon-roton spectrum may persist above the critical temperature.

\section{THEORY}

The method of choice for studying the strongly interacting quantum liquid ${ }^{4} \mathrm{He}$ is the Jastrow-Feenberg theory. The theory is microscopic in the sense that it begins with the best available representation of the many-body Hamiltonian,

$$
H=\sum_{i}\left[-\frac{\hbar^{2}}{2 m} \nabla_{i}^{2}+U_{\mathrm{sub}}\left(\mathbf{r}_{i}\right)\right]+\sum_{i<j} V\left(\left|\mathbf{r}_{i}-\mathbf{r}_{j}\right|\right),
$$


where $V\left(\left|\mathbf{r}_{i}-\mathbf{r}_{j}\right|\right)$ is the He-He interaction [30]. Compared to the bulk liquid, the essential new property that must be dealt with is the breaking of translational invariance and anisotropy of the system. Such symmetry breakings may be induced by the substrate potential $U_{\text {sub }}\left(\mathbf{r}_{i}\right)$, but they may also occur spontaneously like in the formation of quantum liquid droplets [31, 32]. The ground-state theory has been throughly discussed in Ref. 27 and the corresponding excited-state theory is covered in Ref. 23, therefore we only need to review the key points.

The ground-state wave function

$$
\begin{aligned}
\Psi_{0}\left(\mathbf{r}_{1}, \ldots, \mathbf{r}_{N}\right)= & \exp \frac{1}{2}\left[\sum_{i} u_{1}\left(\mathbf{r}_{i}\right)+\sum_{i<j} u_{2}\left(\mathbf{r}_{i}, \mathbf{r}_{j}\right)\right. \\
& \left.+\sum_{i<j<k} u_{3}\left(\mathbf{r}_{i}, \mathbf{r}_{j}, \mathbf{r}_{k}\right)+\ldots\right]
\end{aligned}
$$

is written in Jastrow-Feenberg form; the $n$-body correlation functions $u_{n}\left(\mathbf{r}_{1}, \ldots, \mathbf{r}_{n}\right)$ are determined by functional minimization of the energy. The theory yields the ground-state energetics and structure of the liquid and provides the raw material for investigating excitations.

To introduce excitations to the system one applies a small, time-dependent perturbation that momentarily drives the quantum liquid out of its ground state. This causes the $n$-body correlations in the wave function presented in Eq. (2) to acquire time dependence, hence the excited state has the form

$$
|\Psi(t)\rangle=\frac{e^{-i E_{0} t / \hbar} e^{\frac{1}{2} \delta U(t)}\left|\Psi_{0}\right\rangle}{\left[\left\langle\Psi_{0}\left|e^{\Re e \delta U(t)}\right| \Psi_{0}\right\rangle\right]^{1 / 2}}
$$

with the excitation operator

$$
\delta U(t)=\sum_{i} \delta u_{1}\left(\mathbf{r}_{i} ; t\right)+\sum_{i<j} \delta u_{2}\left(\mathbf{r}_{i}, \mathbf{r}_{j} ; t\right)+\ldots
$$

The time-dependent correlation functions $\delta u_{n}\left(\mathbf{r}_{1}, \ldots, \mathbf{r}_{n} ; t\right)$ are determined by an action principle 33.

$$
\delta \int_{t_{0}}^{t_{1}} d t\left\langle\Psi(t)\left|H-i \hbar \frac{\partial}{\partial t}+U_{\text {ext }}(t)\right| \Psi(t)\right\rangle=0,
$$

where $U_{\text {ext }}(t)$ is the weak external potential driving the excitations.

Linearizing the equations of motion for the wave function $|\Psi(t)\rangle=\left|\Psi_{0}\right\rangle+\delta|\Psi(t)\rangle$ and calculating the timedependent component of the transition density $\delta \rho(\mathbf{r}, t)=$ $\left\langle\Psi_{0}|\hat{\rho}(\mathbf{r})| \delta \Psi(t)\right\rangle+$ c.c. allows us to calculate the densitydensity response function $\chi\left(\mathbf{r}, \mathbf{r}^{\prime} ; \omega\right)$ defined via

$$
\delta \rho(\mathbf{r}, \omega)=\int d^{3} r^{\prime} \chi\left(\mathbf{r}, \mathbf{r}^{\prime} ; \omega\right) U_{\mathrm{ext}}\left(\mathbf{r}^{\prime}, \omega\right) .
$$

Once the response function is known, one can apply the fluctuation-dissipation theorem to find the dynamic structure function,

$$
S\left(\mathbf{r}, \mathbf{r}^{\prime} ; \omega\right)=-\frac{1}{\pi} \Im m \mathcal{X}\left(\mathbf{r}, \mathbf{r}^{\prime} ; \omega\right) .
$$

To obtain the dynamic structure function measured by, e.g., neutron scattering, one has to project $S\left(\mathbf{r}, \mathbf{r}^{\prime}, \omega\right)$ onto plane waves:

$$
S(\mathbf{k}, \omega)=\int d \mathbf{r} d \mathbf{r}^{\prime} e^{i \mathbf{k} \cdot\left(\mathbf{r}-\mathbf{r}^{\prime}\right)} S\left(\mathbf{r}, \mathbf{r}^{\prime} ; \omega\right) .
$$

Up to this points the formulas are valid for any geometry. Since the systems with slit or slab geometry under consideration here are translationally invariant only in the $(x-y)$ plane, but not in the $z$ direction, only the momentum transfer $\mathbf{k}_{\|}$parallel to the film or slab is a good quantum number. In this case, we have

$$
S(\mathbf{k}, \omega)_{\mathrm{film}}=\int d z d z^{\prime} e^{i \mathbf{k}_{\perp}\left(z-z^{\prime}\right)} S\left(\mathbf{k}_{\|}, z, z^{\prime} ; \omega\right) .
$$

The truncation of the sequence of fluctuating correlations $\delta u_{n}$ in Eq. (4) defines the level of approximation in which we treat the excitations. The excitation spectrum can be quite well understood $34,35,36$ by retaining only the time-dependent one- and two-body terms in the excitation operator (4). The two-body terms $\delta u_{2}\left(\mathbf{r}_{1}, \mathbf{r}_{2} ; t\right)$ describe the interaction of two excitations. The simplest non-trivial implementation of the theory leads to a density-density response function of the form 23.

$$
\begin{aligned}
& \chi\left(\mathbf{r}, \mathbf{r}^{\prime}, \omega\right)= \\
& \sqrt{\rho(\mathbf{r})} \sum_{s t} \phi^{(s)}(\mathbf{r})\left[G_{s t}(\omega)+G_{s t}(-\omega)\right] \phi^{(t)}\left(\mathbf{r}^{\prime}\right) \sqrt{\rho\left(\mathbf{r}^{\prime}\right)}
\end{aligned}
$$

where the $\phi^{(s)}(\mathbf{r})$ are Feynman excitation functions, and

$$
G_{s t}(\omega)=\left[\hbar\left[\omega-\omega_{s}+i \epsilon\right] \delta_{s t}+\Sigma_{s t}(\omega)\right]^{-1}
$$

the phonon propagator. The fluctuating pair correlations give rise to the self energy [23],

$$
\Sigma_{s t}(\omega)=\frac{1}{2} \sum_{m n} \frac{V_{m n}^{(s)} V_{m n}^{(t)}}{\hbar\left(\omega_{m}+\omega_{n}-\omega\right)} .
$$

Here, the summation is over the Feynman states $m, n$; they form a partly discrete, partly continuous set due to the inhomogeneity of the liquid. The expression for the three-phonon coupling amplitudes $V_{m n}^{(s)}$ can be found in Ref. 23. This self energy renormalizes the Feynman "phonon" energies $\omega_{n}$, and adds a finite lifetime to states that can decay to two lower-energy modes. The resulting density-density response function has the structure of a Brillouin-Wigner (BW) perturbation formula. The approximate form of the self energy given in Eq. (12) is also closely related to the one obtained using the theory of correlated basis functions (CBF) 34, 35]. As a final refinement to the theory, we scale the Feynman energies 
$\omega_{n}$ appearing in the energy denominator of the self energy given in Eq. (12) such that the roton minimum of the spectrum used in the energy denominator of Eq. (12) agrees roughly with the roton minimum predicted by the calculated $S(\mathbf{k}, \omega)$. This is just a computationally simple way of adding the self energy correction to the excitation energies in the self energy itself. We shall use this scaled CBF-BW approximation for the numerical parts of this paper.

A direct characterization of each mode is obtained by computing the transition density

$$
\delta \rho(\mathbf{r} ; t)=\left\langle\Psi_{0}|\hat{\rho}(\mathbf{r})| \delta \Psi(t)\right\rangle+\text { c.c. }
$$

and the transition current

$$
\delta \mathbf{j}(\mathbf{r} ; t)=\left\langle\Psi_{0}|\hat{\mathbf{j}}(\mathbf{r})| \delta \Psi(t)\right\rangle+\text { c.c. }
$$

where $\hat{\rho}(\mathbf{r})$ and $\hat{\mathbf{j}}(\mathbf{r})$ are the familiar one-body density and current operators. The transition density shows the density change (arbitrary amplitude) and the transition current the flow pattern of atoms in the mode.

\section{RESULTS}

We have modeled the confined quantum liquid by ${ }^{4} \mathrm{He}$ between two planar substrates. The distance between the substrate planes in our calculations is $40 \AA$ which is not too far from the diameter of aerogel strands and an intermediate value between the pore or channel diameters in Vycor $(d \sim 70 \AA)$ and Geltech $(d \sim 25 \AA)$. We assume translational invariance in a plane parallel to the surface. This is, of course, not exactly true in the above mentioned materials. However, our assumption should only change the details of the excitations spectrum at long wave lengths that are comparable to the pore size, whereas rotons arise from short range correlations.

We have computed the dynamic structure function as a function of the scattering angle for a thick film and a filled gap. The density profiles for these two situations are shown in Fig. 1] The interaction of ${ }^{4} \mathrm{He}$ particles with the walls is described by the usual 3-9 potential obtained from averaging Lennard-Jones potentials over a half space. This is valid if the walls are smooth and, hence, $U_{\text {sub }}(\mathbf{r})$ is a function of one coordinate only. Our approximation is legitimate because the lateral structure of surface is smoothed out by the first solid layer of helium atoms. The 3-9 potential used in this work is derived from the silicon-helium interaction [37]; we have supplemented this potential by a $4-10$ potential due to averaging Lennard-Jones 6-12 potentials for the ${ }^{4} \mathrm{He}^{-}{ }^{4} \mathrm{He}$ interaction over a plane. Thus, our substrate potential has the form

$$
U_{\mathrm{sub}}(z)=U_{3}(z-z 0)+U_{4}(z)
$$

where $U_{3}(z)$ is the common 3-9 potential 37.

$$
U_{3}(z)=\left[\frac{4 C_{3}^{3}}{27 D^{2}}\right] \frac{1}{z^{9}}-\frac{C_{3}}{z^{3}}
$$

with a well-depth $D=128 \mathrm{~K}$ and a range $C_{3}=$ $2000 \mathrm{~K}^{3}$. The potential $U_{4}(z)$ has the form

$$
U_{4}(z)=4 \pi \epsilon \rho_{1} \sigma^{2}\left[\frac{1}{5}\left(\frac{\sigma /}{z}\right)^{10}-\frac{1}{2}\left(\frac{\sigma}{z}\right)^{4}\right] .
$$

Here, $\epsilon=10.22 \mathrm{~K}$ and $\sigma=2.556 \AA$ are the usual deBoerMichels parameters for the helium-helium interaction. The areal density of the solid monolayer was taken to be $\rho_{1}=0.07 \AA^{2}$ and a thickness $z_{0}=3.3 \AA$. The picture is consistent values for hectorite gaps [38], the areal density is somewhat lower than the one of the second solid layer on graphite 39].

The helium film considered here is thick enough to support layer rotons; ripplons will also appear for the film model which has a free surface. In the filled gap case the amount of helium between the Si planes was chosen to correspond the filling of an aerogel sample surrounded by bulk liquid in equilibrium. The chemical potential of the confined liquid and that of a reservoir of bulk liquid turned out to be equal $(\mu \approx-7.2 \mathrm{~K})$ at $n=0.85 \AA^{-2}$. For comparison, a $40 \AA$ thick slice of bulk liquid at the equilibrium density $0.02185 \AA^{-3}$ would correspond to the coverage $n=0.87 \AA^{-2}$.

Some excitations modes are bulk-like, these have no significant angle dependence. In the bulk liquid the roton energy decreases and the roton minimum moves to higher momenta as the density increases. Traces of this effect are also visible in the $3 \mathrm{D}$ roton: the roton energy is above it's equilibrium bulk value because of the low density regions between the layers.

Layer modes, on the other hand, are entirely different. In films, layer rotons propagate mainly in the highest density liquid layer closest to the substrate, hence their motion is well confined to two dimensions and their propagation direction is always parallel to the surface. Their energy has a parabolic minimum at a fixed wave vector $\mathbf{k}_{\|, 0}$ in the plane parallel to the surface. Upon changing the orientation of the surface also the in-plane component of the total wave vector transfer changes. As a result, the location of the layer roton minimum shifts.

We will show our results for the dynamic structure function as gray-scale plots. In these maps of the dynamic structure function, darker areas correspond to higher value of $S(k, \omega)$. To emphasize low-intensity regions we use the scale $S(k, \omega)^{1 / 4}$, the same gray scale will be used throughout this work. To facilitate plotting we have also introduced a $0.05 \mathrm{~K}$ Lorentzian broadening of the structure functions. We measure the scattering angle from the substrate plane, so $\theta=0$ corresponds to grazing angle and $\theta=90$ degrees is scattering perpendicular to the substrate plane.

\section{A. Thick ${ }^{4}$ He films on silicon}

Fig. 2] shows the dynamic structure function of a thick ${ }^{4} \mathrm{He}$ film on Si for scattering angles $\theta=0$ and $\theta=90$ de- 


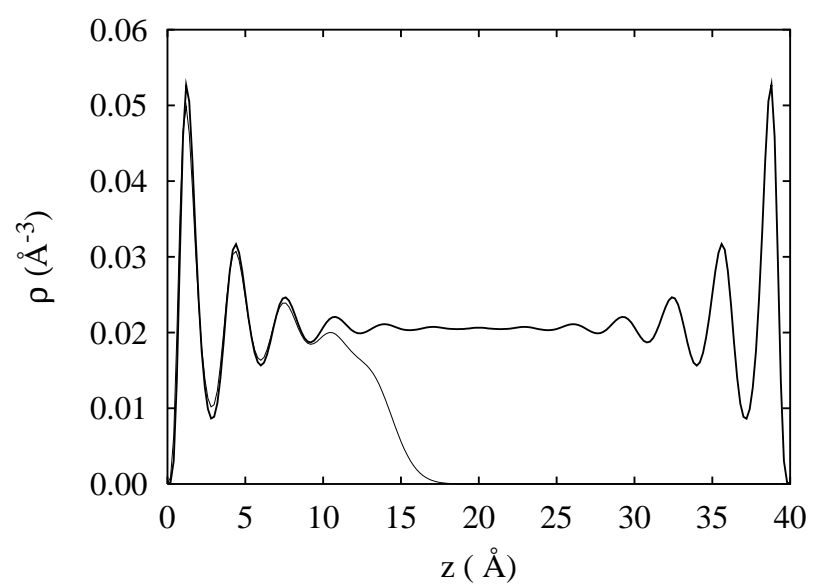

FIG. 1: The density profiles of the film and the filled gap used in this work. The coverages of the film is $0.3 \AA^{-2}$, and the filled gap has 0.85 atoms per $\AA^{2}$.

grees. The former shows a ripplon mode with dispersion $\hbar \omega(k) \sim k^{3 / 2}$, and also two layer rotons below the bulk roton are clearly distinguishable. Perpendicular scattering shows a discrete set of low-energy dispersionless modes, but some strong resonances appear also above the continuum limit $-\mu=7.7 \mathrm{~K}$. In the perpendicular case the familiar phonon-roton form is still recognizable, but beside it there are secondary maxima, which are roughly evenly spaced in $\mathbf{k}_{\perp}$. These strands appear because the projection onto plane waves in Eq.(9) gives contributions to multiple perpendicular momenta $\mathbf{k}_{\perp}$, and the dynamic structure function shows features coming from the Fourier transform of the density profile. A detailed description of how the strands appear was recently given in Ref. 40 .

Randomly oriented surfaces give rise to an angular averaged response. Fig. B shows such a theoretical dynamic structure function, where contributions from several scattering angles between 0 and 90 degrees have been added. While the contributions from bulk-like rotons add up to a single curve, the ones from $2 \mathrm{D}$ modes do not. The low$k$ region shows faints steps at about $\hbar \omega=0 \mathrm{~K}, 1.9 \mathrm{~K}$, $4.4 \mathrm{~K}, 6.0 \mathrm{~K}$ etc., reminiscent of the states turning dispersionless as the angle increases; hence the fan-like structures. The strongest of these comes from the ripplons, and is probably the only one that can be in experiments. As mentioned earlier, the layer roton minimum shifts to higher total momentum transfers as the scattering angle increases. This has not been seen in neutron scattering experiments, which show a clear parabolic dispersion for the layer modes. The parallel direction gives the the strongest signal in neutron scattering, and we can only assume that the contribution from other angles in the raw data is simply too small to give any information to be inverted to $S(k, \omega)$.

Fig. 4 shows a fully angle-averaged dynamic structure function at $k=1.8 \AA^{-1}$, near the roton minimum. Angle
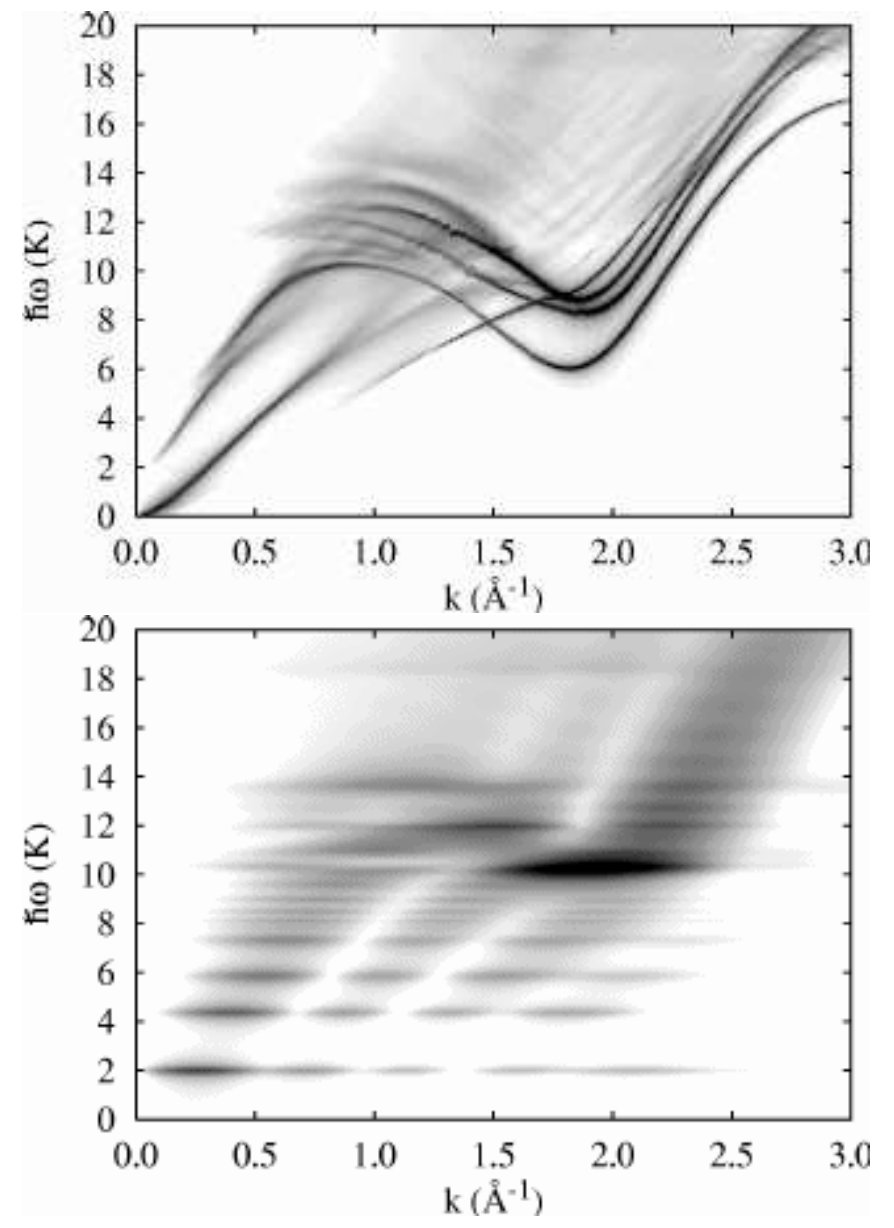

FIG. 2: The dynamic structure function of a thick ${ }^{4} \mathrm{He}$ film on a Si surface (coverage $n=0.3 \AA^{-2}$ ) at grazing angle (upper panel) and perpendicular to the substrate (lower panel).

averaging causes the layer mode peaks to become asymmetric, while it has no effect on the bulk-like modes. The asymmetry is clearly visible in the first-layer peak, which has a broad tail on the high-energy side. This broadening is just due to summing up of the multiple layer roton parabola, which show as separate curves in Fig. 3 A direct identification of the modes is given by the transition density and current, shown for the thick film in Fig. 5 for $\theta=0$. The scale on the right corresponds to the transition current, depicted as a vector field, for the duration of one oscillatory cycle which takes about $48 \times \hbar \omega \mathrm{K}^{-1}$ picoseconds. The lowest energy mode for grazing angle is a layer roton, which involves atoms in the layer closest to the substrate. The next mode is loosely confined to the second layer, but subsequent modes spread throughout the liquid as there are no more well-separated layers available. From the third mode on the excitations are three dimensional, as well as they can be in a $15 \AA$ thick film.

The situation in the perpendicular scattering is completely different. The lowest panel of Fig. [6 shows the mode at $\hbar \omega=1.9 \mathrm{~K}$, which already has one node in the 


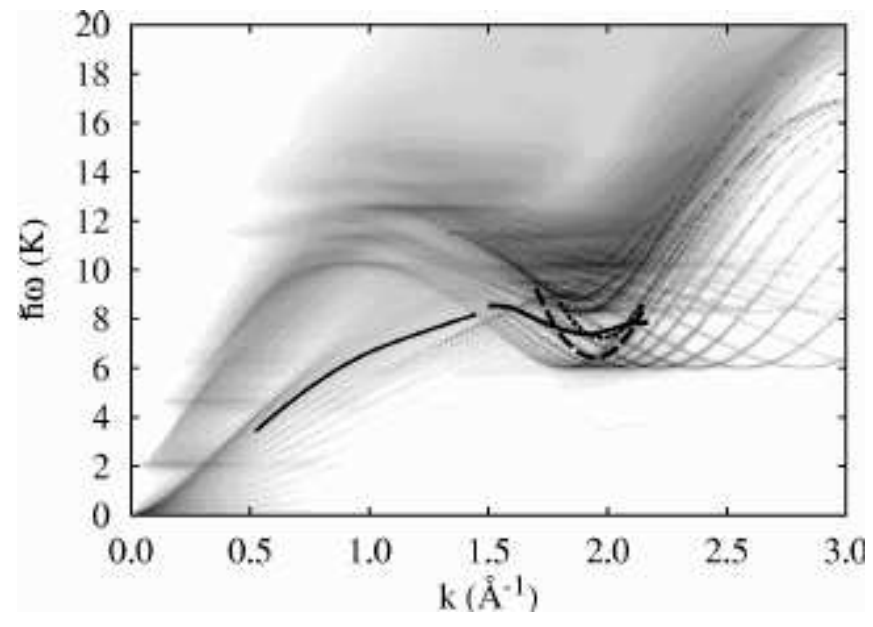

FIG. 3: The dynamic structure function of a thick film on a Si surface (coverage $n=0.3 \AA^{-2}$ ). The plot shows the sum of contributions at angles between 0 and 90 degrees with 5 degree steps. The dashed lines show the experimental layer roton dispersion for aerogel (upper curve) and Vycor (lower curve) by Plantevin et al. 10]; The solid curve shows the layer roton and ripplon data from Ref. 41 .

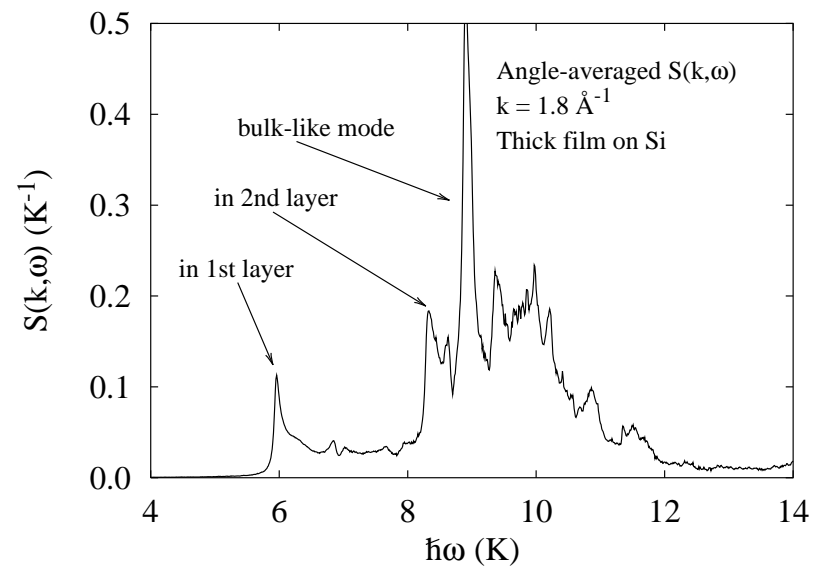

FIG. 4: The fully angle-averaged $S(k, \omega)$ of a thick film at $k=1.8 \AA^{-1}$. The coverage is $0.3 \AA^{-2}$.

transition density. Particle number conservation forbids a nodeless mode, so the first real excitation is one where the liquid oscillates with respect to the substrate.

Fig. 6] can be looked upon as a pictorial view of quantum evaporation. Higher energy modes involve motion of atoms near the surface, and finally modes with energy above minus the chemical potential (now $\mu=-7.7 \mathrm{~K}$ ) are energetic enough to kick out atoms to continuum states.

\section{B. ${ }^{4}$ He filling space between two silicon surfaces}

Fig. [7 shows the energy and pressure in the case, where helium fills the space between the substrate walls. The equilibrium density of the filled gap by itself would be
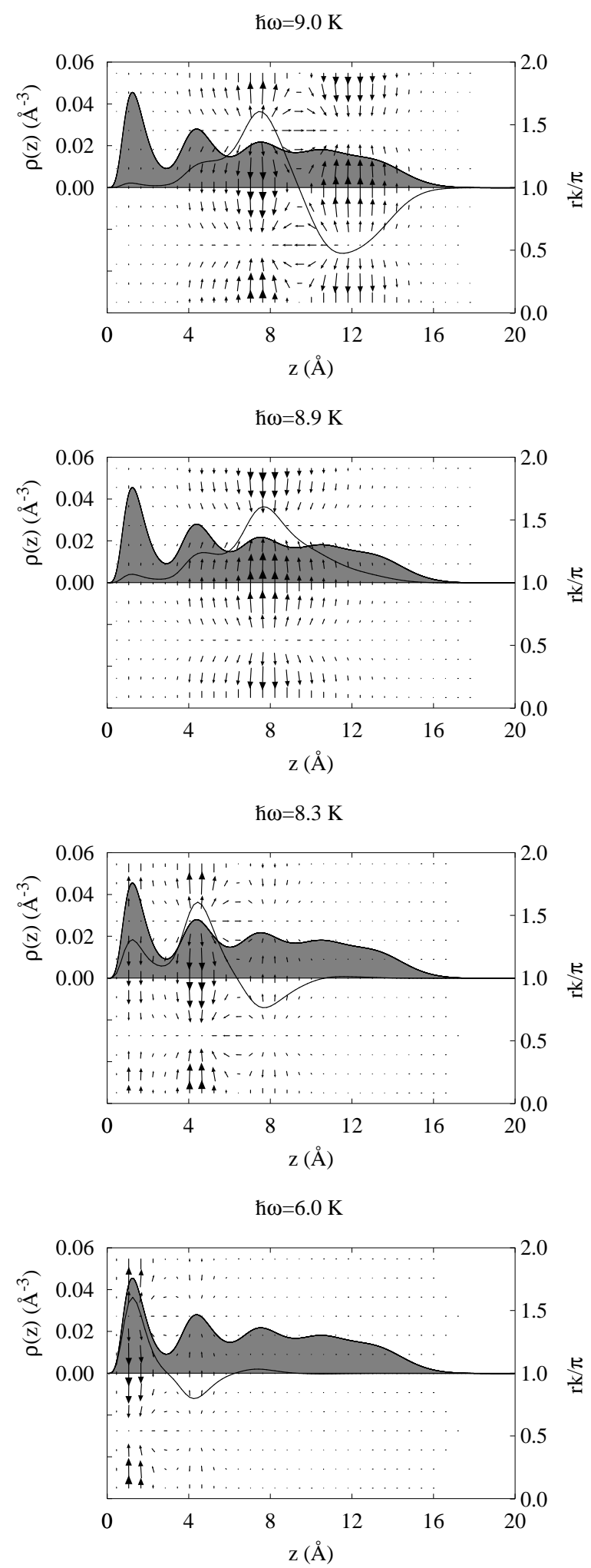

FIG. 5: Transition density (thick line) and transition current (arrows; right scale) for the low-energy modes at $k=1.8 \AA^{-1}$ for a thick film on Si substrate and scattering parallel to the surfaces, $\theta=0$. The grayscale plot shows the density profile. Excitations energies are indicated in the figures, the lowest panel corresponds to lowest energy. 

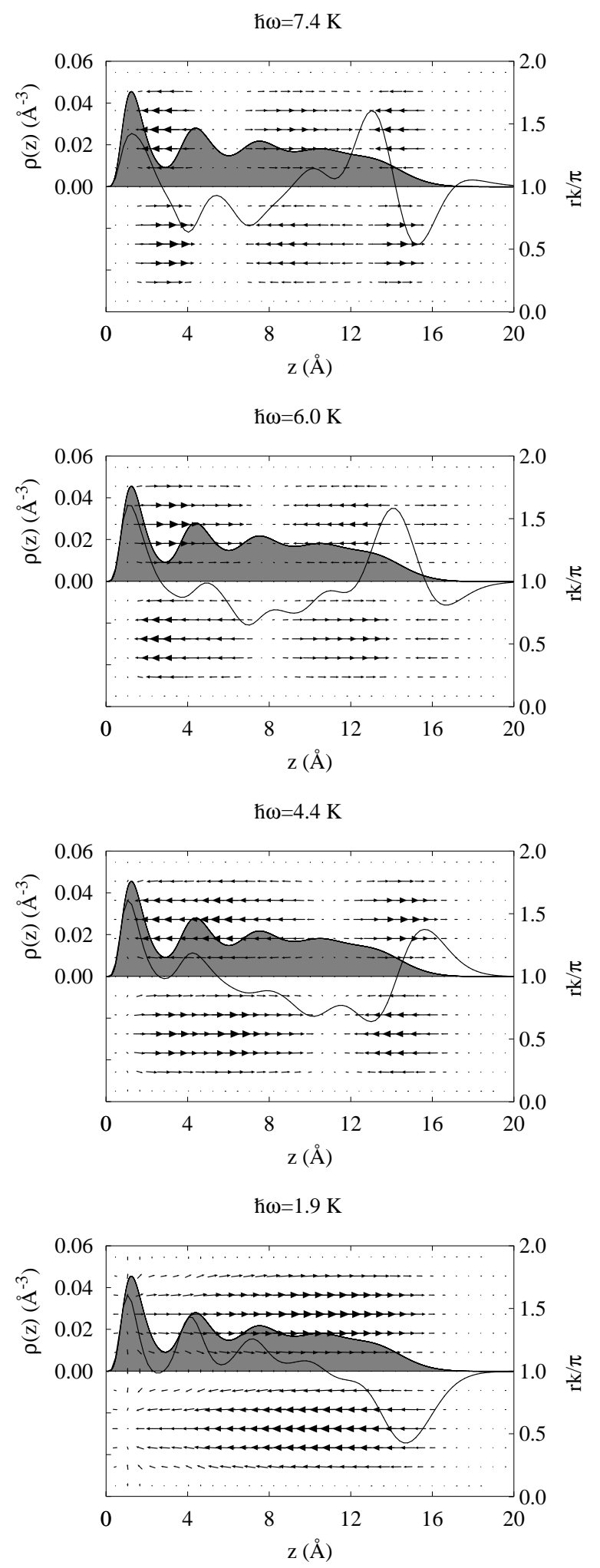

FIG. 6: Same as Fig. 國 for $\theta=90$ (specular scattering).

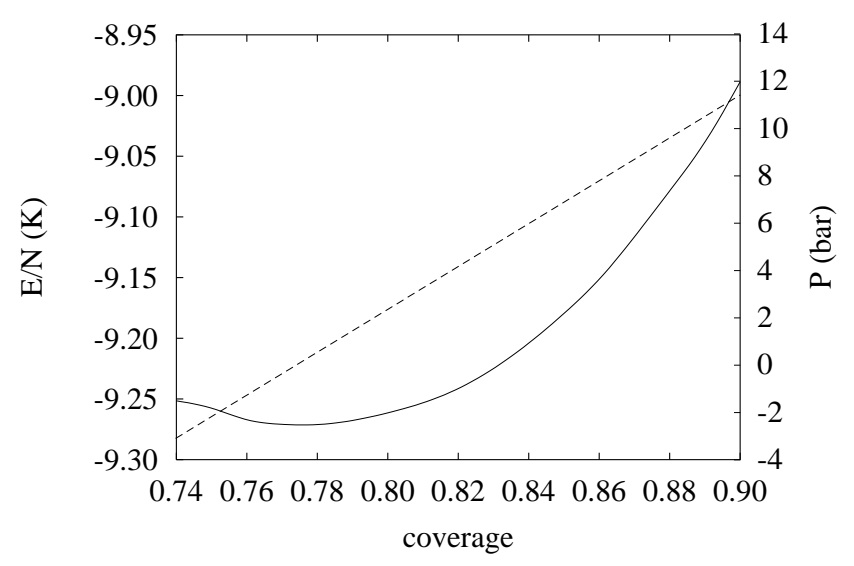

FIG. 7: The total energy (solid line, left scale) and pressure (dashed line, right scale) of a helium filled $40 \AA$ wide gap as a function of coverage. Liquid in the middle of the gap becomes unstable at about $0.68 \AA^{-2}$ indicating the formation of two separate thick films on both walls.

at about $\rho=0.78 \AA^{-2}$, but the liquid is in balance with an external bulk ${ }^{4} \mathrm{He}$ reservoir at $\rho=0.85 \AA^{-2}$. Below $0.68 \AA^{-2}$ the liquid is no longer translationally invariant parallel to the walls, and the instability in the liquid solution is an indication of capillary condensation. At low density liquid forms two films covering both surfaces. In the filled gap case, if the density of helium between the walls is further increased, one observes a sequence of layering transitions: the number of helium layers increases. This filling scenario has been discussed in detail in Ref. [28].

The (partially) angle averaged $S(k, \omega)$ in Fig. 8 is qualitatively different from the film case result in Fig. [3 only in the low-momentum region. There is now only one strong maximum corresponding to bulk phonons on top of a faint step structure. The steps are a remainder of the discrete states in the perpendicular or nearly perpendicular directions. The angle averaged $S(k, \omega)$ near the roton wave vector is quite similar to the one in the thick film case, depicted in Fig. 目

The transition densities and currents are shown in Figs. 9 and 10 for scattering angles 0 and 90 degrees, respectively. At grazing angle the low-energy excitation reside in the layers near the substrate, and pairs of modes corresponding to in- and out-of-phase oscillations are degenerate. The modes with even number of oscillations have their counterparts in the case of two films on both walls, as one can see by comparing Figs. [6 and 10] The lowest mode depicted in Fig. 10 with odd number of oscillations have liquid oscillating back and forth in the middle of the gap, and with decreasing density this oscillation is finally able to divide the system into two films. The point where this mode becomes soft is the spinodal instability of the filled case. 


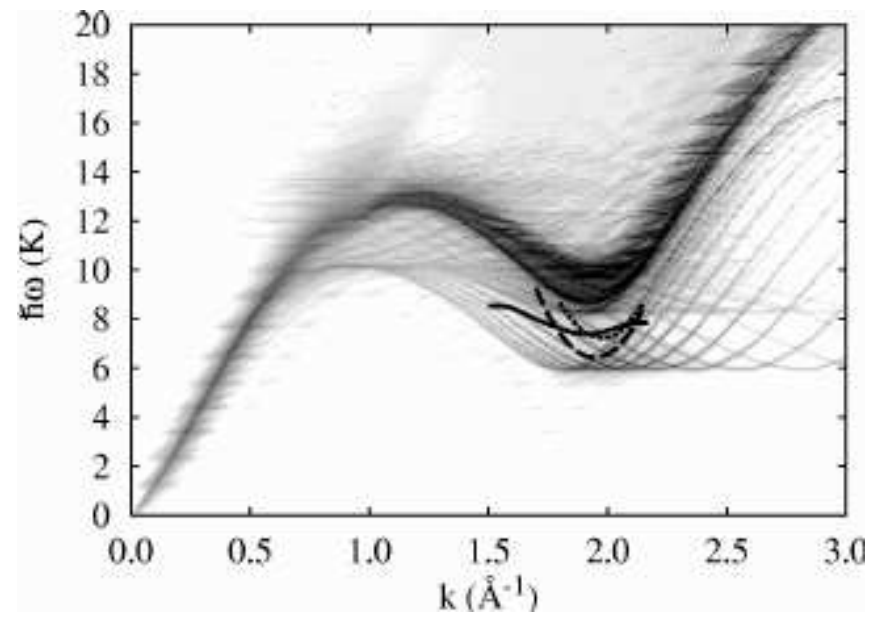

FIG. 8: The dynamic structure function of the filled gap. The plot shows the sum of contributions at angles between 0 and 90 degrees in 5 degree steps. The calculated layer roton shifts to higher $k$ as a function of angle. The dashed lines show the experimental layer roton dispersion for aerogel (upper curve) and Vycor (lower curve) by Plantevin et al. 10]; the solid curve shows results from Ref. 41 .

\section{CONCLUSIONS}

In order to model ${ }^{4} \mathrm{He}$ confined in materials like aerogel, Vycor or Geltech, we have calculated the energetics, the density profile and the dynamical properties of thick ${ }^{4} \mathrm{He}$ film on Si substrate and between two substrate planes. The simulation of confinement in spherical pores, which is perhaps the model that is closest to reality, is also feasible with our techniques, but computationally less efficient. For the excitations near the roton wave vector, where the most interesting phenomena are occurring, the use of a simplified planar geometry should not make much difference because the roton wave length is much shorter than any lengthscale on which the lateral structure of the aerogel changes. The two-wall geometry has actually been used in specific heat measurements by Gasparini and collaborators [42, 43, 44, 45], but the distance between the silicon planes was a couple of magnitudes larger than in our model. For a large wall distance the mid section of the liquid is essentially unaware of the substrate. Thus one would have a thick region of basic bulk helium, which would show up almost exclusively in the dynamic structure function.

The results for thick helium films are relevant for large pores at low filling, when opposite walls are approximately noninteracting and the curvature of the liquid can be ignored. We have not considered the possibility of unevenly filled pores or a distribution pore sizes, because the characteristics of porous systems are very material dependent, and our aim was to discuss phenomena that are universal in such materials.

There are presently two sets of data for the layer roton, those of Refs. 10 and of 41 . When comparing theory and experiment, two aspects are of interest: One is the
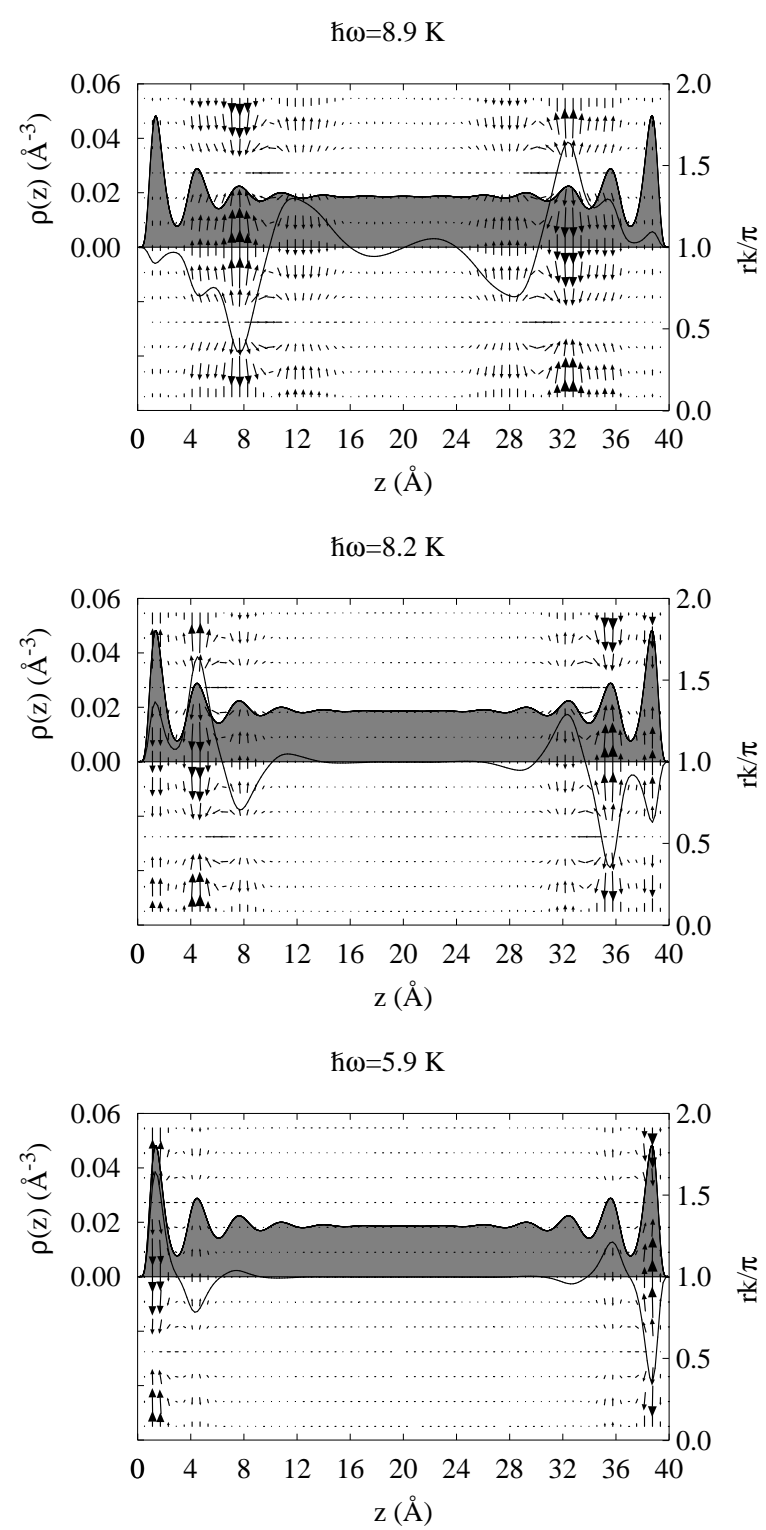

FIG. 9: Transition density (thick line) and transition current (arrows; right scale) in the low-energy modes at $k=1.8 \AA^{-1}$ in the helium filled gap at $n=0.85 \AA^{-2}$ for grazing angle. The grayscale plot shows the density profile. The transition density has arbitrary scale. Excitations energies are indicated in the figures, lowest panel corresponds to lowest energy. Due to the symmetric density profile there is pairwise degeneracy of modes corresponding to in-phase and out-of-phase oscillation between the left and the right side; we show here only the latter ones.

energy of the layer roton, and the other one the dispersion curve. As can be seen from the measurements of Ref. 10 and 41 shown in Figs. 3 and 8 as well as from other measurements 25, 26], that the energy of the layer roton depends sensitively on the nature of the substrate. This is expected because different substrates will lead to different densities of the first liquid layers and, hence, to 
$\hbar \omega=4.7 \mathrm{~K}$

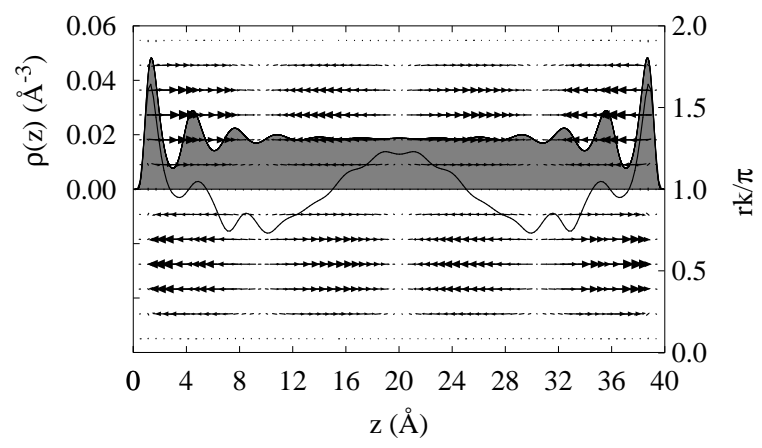

$\hbar \omega=3.5 \mathrm{~K}$

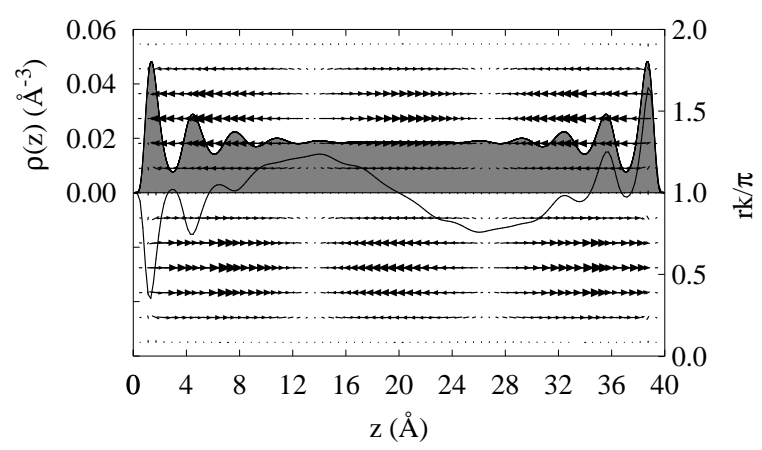

$\hbar \omega=2.3 \mathrm{~K}$

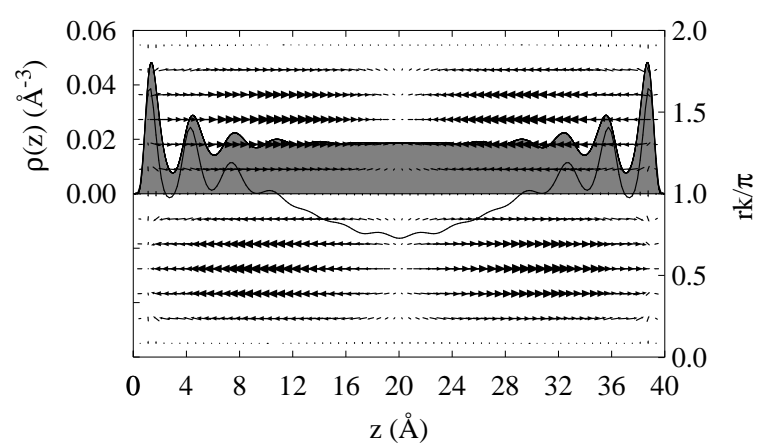

$\hbar \omega=1.2 \mathrm{~K}$

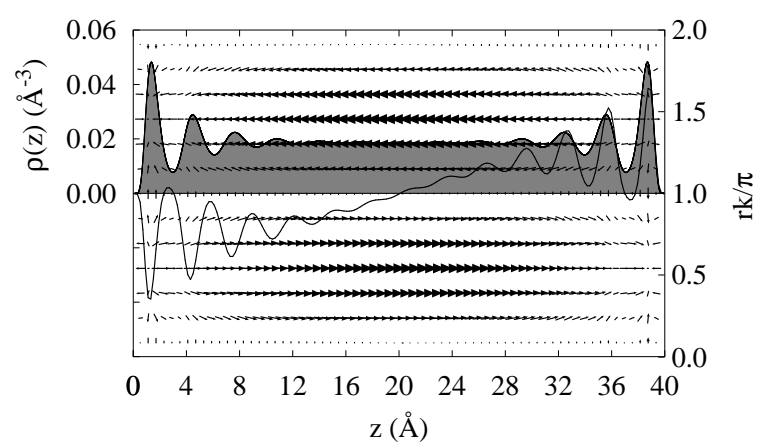

FIG. 10: Same as Fig. 9 for the 90 degree angle (specular scattering), except that now there is no degeneracy and we show all low-energy modes. different phonon-roton spectra. Indeed, the dependence is so sensitive that it might be possible to determine the strength of the substrate potential from the energy of the roton gap.

The second aspect is the shape of the dispersion curve. The measurements of Ref. 41 show a significantly smaller curvature of the dispersion curve than those of the other experiments, but they agree better with our calculations. Keeping the extreme difficulty of extracting these data from neutron scattering spectra in mind, we tend towards the view that the experiments of Ref. 41 provide a more accurate description. This is so for two reasons: First, the curvature of the roton in purely two-dimensional ${ }^{4} \mathrm{He}$ is smaller than that obtained in Ref. 10, and second, as shown in Figs. B and 8 the angular averaging leads to a further broadening.

\section{Acknowledgments}

This work was supported by the Austrian Science Fund (FWF) under project P12832-TPH. One of us (VA) would like to thank the Department of Physics at the Technical University of Denmark, where part of this work was done, for hospitality. 
[1] W. M. Snow and P. E. Sokol, J. Low. Temp. Phys. 80, 197 (1990).

[2] J. de Kinder, G. Goddens, and R. Millet, Z. Phys. B 95, 511 (1994).

[3] R. M. Dimeo, P. E. Sokol, D. W. Brown, C. R. Anderson, W. G. Stirling, M. A. Adams, S. H. Lee, C. Rutiser, and S. Komarneni, Phys. Rev. Lett. 79, 5274 (1997).

[4] R. M. Dimeo, P. E. Sokol, C. R. Anderson, W. G. Stirling, and M. A. Adams, J. Low Temp- Phys. 113, 369 (1998).

[5] P. E. Sokol, M. R. Gibbs, W. G. Stirling, R. T. Azuah, and M. A. Adams, Nature 379, 616 (1996).

[6] M. R. Gibbs, P. E. S. W. G. Stirling, R. T. Azuah, and M. A. Adams, J. Low Temp. Phys. 107, 33 (1997).

[7] O. Plantevin, B. Fåk, H. R. Glyde, J. Bossy, and J. R. Beamish, Phys. Rev. B 57, 10775 (1998).

[8] H. R. Glyde, B. Fåk, and O. Plantevin, J. Low Temp. Phys. (1998).

[9] B. Fåk, O. Plantevin, and H. R. Glyde, Physica B 276278, 806 (2000).

[10] O. Plantevin, B. Fåk, H. R. Glyde, N. Mulders, J. Bossy, G. Coddens, and H. Schober, Phys. Rev. B 63, 224508 (2001).

[11] O. Plantevin, H. R. Glyde, B. Fåk, J. Bossy, F. Albergamo, N. Mulders, and H. Schober, Phys. Rev. B 65, 224505 (2002).

[12] C. R. Anderson, K. H. Andersen, J. Bossy, W. G. Stirling, R. M. Dimeo, P. E. Sokol, J. C. Cook, and D. W. Brown, Phys. Rev. B 59, 13588 (1999).

[13] H. J. Lauter, H. Godfrin, and P. Leiderer, J. Low Temp. Phys. 87, 425 (1992).

[14] H. J. Lauter, H. Godfrin, V. L. P. Frank, and P. Leiderer, Phys. Rev. Lett. 68, 2484 (1992).

[15] C. Bäuerle, B. Clements, H. Godfrin, H. J. Lauter, P. Leiderer, and V. Passiouk-Lauter, Tech. Rep. 6-01-160, Institut Laue-Langevin (ILL) (1996).

[16] H. Godfrin, J. Klier, V. Lauter-Pasyuk, H. Lauter, and P. Leiderer, Tech. Rep. 6-01-174, Institut Laue-Langevin (ILL) (1998).

[17] H. J. Lauter, V. L. P. Frank, H. Godfrin, and P. Leiderer, Elementary Excitations in Quantum Fluids (SpringerVerlag, Berlin, 1989), vol. 79 of Springer Series in Solid State Sciences, p. 99.

[18] H. Godfrin, V. L. P. Frank, H. J. Lauter, and P. Leiderer, in Phonons 89, edited by S. Hunklinger, W. Ludwig, and G. Weiss (World Scientific, Singapore, 1989), p. 904.

[19] H. J. Lauter, H. Godfrin, V. L. P. Frank, and P. Leiderer, Excitations in two-dimensional and three-dimensional quantum fluids (Plenum, New York, 1991), vol. 257 of NATO Anvanced Study Institute, Series B: Physics, p. 419.

[20] H. J. Lauter, H. Godfrin, V. L. P. Frank, and P. Leiderer, Phys. Rev. Lett. 68, 2484 (1992).

[21] B. E. Clements, H. Godfrin, H. Lauter, P. Leiderer, E. Krotscheck, and C. J. Tymczak, J. Low Temp. Phys. (rapid communications) 102, 1 (1996).

[22] B. E. Clements, H. Godfrin, E. Krotscheck, H. J. Lauter,
P. Leiderer, V. Passiouk, and C. J. Tymczak, Phys. Rev. B 53, 12242 (1996).

[23] B. E. Clements, E. Krotscheck, and C. J. Tymczak, Phys. Rev. B 53, 12253 (1996).

[24] B. E. Clements, E. Krotscheck, and C. J. Tymczak, J. Low Temp. Phys. 107, 387 (1997).

[25] H. R. Glyde, O. Plantevin, B. Fåk, G. Coddens, P. S. Danielson, and H. Schober, Phys. Rev. Lett. 84, 2646 (2000).

[26] B. Fåk, O. Plantevin, H. R. Glyde, and N. Mulders, Phys. Rev. Lett. 85, 3886 (2000).

[27] B. E. Clements, J. L. Epstein, E. Krotscheck, and M. Saarela, Phys. Rev. B 48, 7450 (1993).

[28] V. Apaja and E. Krotscheck, Phys. Rev. B 64, 134503 (2001).

[29] M. Boninsegni and H. R. Glyde, J. Low Temp. Phys. 112, 251 (1998).

[30] R. A. Aziz, F. R. W. McCourt, and C. C. K. Wong, Molec. Phys. 61, 1487 (1987).

[31] S. A. Chin and E. Krotscheck, Phys. Rev. B 52, 10405 (1995).

[32] E. Krotscheck and R. Zillich, J. Chem. Phys. 22, 10161 (2001).

[33] P. Kramer and M. Saraceno, Geometry of the TimeDependent Variational Principle in Quantum Mechanics (Springer, Berlin, 1981), vol. 140 of Lecture Notes in Physics.

[34] H. W. Jackson, Phys. Rev. A 8, 1529 (1973).

[35] C. C. Chang and C. E. Campbell, Phys. Rev. B 13, 3779 (1976).

[36] V. Apaja and M. Saarela, Phys. Rev. B 57, 5358 (1998).

[37] G. Vidali, G. Ihm, H.-Y. Kim, and M. W. Cole, Surface Science Reports 12, 133 (1991).

[38] N. Wada, A. Inoue, H. Yano, and K. Torii, Phys. Rev. B 52, 1167 (1996).

[39] H. J. Lauter, H. Godfrin, V. L. P. Frank, and P. Leiderer, in Excitations in Two-Dimensional and ThreeDimensional Quantum Fluids, edited by A. F. G. Wyatt and H. J. Lauter (Plenum, New York, 1991), vol. 257 of NATO Advanced Study Institute, Series B: Physics, pp. 419-427.

[40] V. Apaja, H. Godfrin, E. Krotscheck, and H. Lauter, J. Low Temp. Phys. 124, 599 (2001).

[41] H. J. Lauter, I. V. Bogoyavlenskii, A. V. Puchov, H. Godfrin, S. Skomorokhov, J. Klier, and P. Leiderer, Applied Physics (Suppl.) A74, S1547 (2002).

[42] I. Rhee, F. M. Gasparini, and D. J. Bishop, Phys. Rev. Lett. 63, 410 (1989).

[43] S. Mehta, M. O. Kimball, and F. M. Gasparini, J. Low Temp. Phys. 113, 435 (1998).

[44] S. Mehta, M. O. Kimball, and F. M. Gasparini, J. Low Temp. Phys. 114, 467 (1999).

[45] M. O. Kimball and F. M. Gasparini, Physica B 284-288, $47(2000)$. 